\title{
Analysis of an Optical Force Sensor for Haptic Applications
}

\author{
Lucas Wan \\ Department of Mechanical Engineering \\ Dalhousie University \\ Halifax, Nova Scotia, Canada \\ lucas.wan@dal.ca
}

\author{
Ya-Jun Pan \\ Department of Mechanical Engineering \\ Dalhousie University \\ Halifax, Nova Scotia, Canada \\ yajun.pan@dal.ca
}

\begin{abstract}
The purpose of this project is to evaluate a midpriced 3-axis optical force sensor for low force applications, such as the validation of haptic devices. By applying a range of static loads, the sensor was calibrated, and the hysteresis, repeatability, and non-linearity of the sensor was analysed. The results of the sensor testing were compared to the manufacturer specifications and to the requirements for using the sensor to measure the force output by a haptic device. Custom components for testing the sensor were designed and 3D printed. The sensitivity of the sensor was found to have deteriorated over time. The sensor exhibited significant hysteresis and non-linearity for low forces, though the results with respect to the nominal capacity agreed with the manufacturer specifications. The sensor was determined to have acceptable results in resolution, accuracy, and repeatability for use in the validation of haptic systems. The sensor was shown to retain a reading of $10 \%$ of the loaded force after it was unloaded, which may invalidate the sensor for use in some applications.
\end{abstract}

\section{INTRODUCTION}

Optical force sensors are a relatively recent technology that have applications in automated manufacturing, medical robotics, and haptic devices. Typical force sensors are strain gauge load cells and piezoelectric force transducers. Load cells have the disadvantages of being rigid and bulky, while piezoelectric transducers are suited for dynamic force measurements. An optical force sensor has the advantage of being a small, deformable, single structure that can measure forces along the $\mathrm{x}, \mathrm{y}$, and $\mathrm{z}$-axes. Optical force sensors are durable and robust because the sensing element is separate from the deforming element.

In [1] a similar 3-axis optical tactile sensor is designed and tested. With applied static loads of up to $4 \mathrm{~kg}$, the sensor was found to have an average deviation of $35 \mathrm{~g}$ and a maximum deviation of $80 \mathrm{~g} \mathrm{(2 \% )}$ from the applied load. A 3-axis optical sensor presented in [2] exhibited a hysteresis of $10 \%$.

A high-end 6-axis strain gauge force sensor (SI-65-5) with an accuracy of $1 \%$ is used in [3] to characterise the force applied by a haptic device. In [4] the difference between the issued force and the output force of a novel haptic device is measured using a high-end 6-axis strain gauge force sensor (ATI Mini 45) with an accuracy of $1.5 \%$ [5].

Before the optical sensor is used for research applications, the specifications given by the manufacturer must be experimentally validated. The calibration, hysteresis, repeatability, and non-linearity of the sensor will be evaluated in the
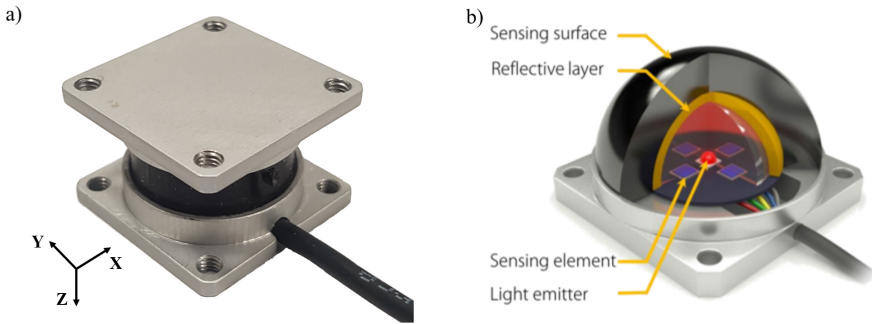

Fig. 1. (a) Flattop optical force sensor with positive axis readings labelled. (b) Cross-section of an Optoforce optical force sensor.

Source: Adapted from [7]

TABLE I

MANUFACTURER SPECIFICATIONS OF OPTICAL FORCE SENSOR SOURCE: ADAPTED FROM [8]

\begin{tabular}{cccc}
\hline & $\mathrm{Z}_{\text {comp }}$ & $\mathrm{Z}_{\text {tens }}$ & $\mathrm{XY}$ \\
\hline Nominal Capacity [N] & 100 & 50 & \pm 10 \\
Resolution [N] & 0.006 & 0.006 & 0.001 \\
Hysteresis & $2 \%$ & $2 \%$ & - \\
Full Scale Non-Linearity & $2 \%$ & $5 \%$ & $2 \%$ \\
\hline
\end{tabular}

following sections. The results will be assessed based on the requirements for using the sensor to validate the transparency of haptic device systems [6].

\section{Methodology}

The sensor used in this paper is a mid-priced flattop Optoforce 3-axis optical force sensor (OMD-20-FG-100N), shown in Fig. 1 (a). It uses an infrared LED light and photodiode light sensors to detect the strain applied to the outer rubber surface. As the outer surface and inner reflective layer is deformed, the reflection of the light changes and the intensity of the light on the sensors fluctuates. A cross-section of the sensor is shown in Fig. 1 (b). The experimental results of resolution, hysteresis, and non-linearity will be compared to the manufacturer's specifications, presented in Table I.

\section{A. Test Setup}

The testing setup of the sensor was designed to require the minimum number of components. The mounting components for the test were 3D printed and designed specifically for the Optoforce flattop optical force sensor. The base component 


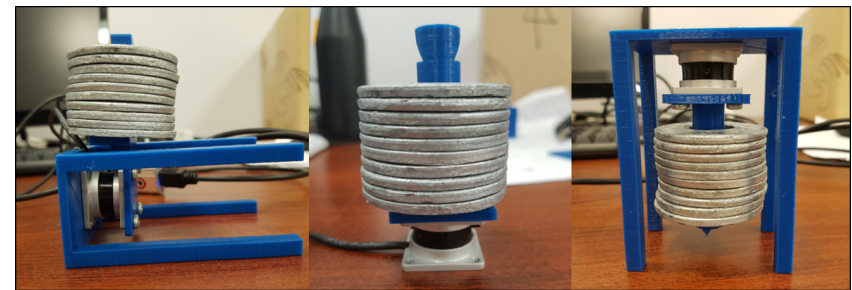

Fig. 2. Test setup for $\mathrm{x}$ and $\mathrm{y}$-axes, $\mathrm{z}$-axis in compression, and $\mathrm{z}$-axis in tension

was used to mount the sensor so that it did not come into contact with the ground as it deformed during the tests. There were two mass supports, one for force in the $\mathrm{z}$-axis and one for force in the $\mathrm{x}$ and $\mathrm{y}$-axes. The $\mathrm{z}$-axis support used a pin to support the mass as the sensor was tested in tension. The testing setup is shown in Fig. 2. A range of static loads were applied by setting ten washers onto the sensor in a specific order, one at a time. A wait time of five seconds was used between the placement of each washer to let the vibrations settle so that a constant force reading could be taken. The average mass of the washers is $30.1 \mathrm{~g} \pm 0.3 \mathrm{~g}$. The total applied load with all ten washers is $2.95 \mathrm{~N}$, which defines the maximum working range in the scope of this paper. According to [6], the required range for a sensor used to validate the system of a haptic device is $\pm 3.3 \mathrm{~N}$. For calibration, repeatability, and non-linearity data acquisition, the sensor was loaded to the maximum working range in both directions of each axis. For the hysteresis data acquisition, the sensor was loaded to the maximum working range and unloaded to zero in both compression and tension in the zaxis. To compare the data to the manufacturer specifications in Table I the maximum hysteresis and non-linearity data percentages are also calculated with respect to the nominal capacities of the sensor. This assumes that the maximum errors occur in the range of $0 \mathrm{~N}$ to $2.95 \mathrm{~N}$, which may result in some inaccuracies in the characterization of the sensor.

\section{B. Data Collection}

The data was collected with a USB DAQ (Data Acquisition) device supplied with the sensor. Optoforce data visualization software was used to monitor the data collection and export the data to a csv file. The data was imported into Matlab where it was processed and analysed. The raw force data is represented as counts, therefore a calibration factor had to be experimentally determined to convert the data into Newtons. In the z-axis, compression produces a positive sensor reading and tension produces a negative sensor reading. The data was sampled at a frequency of $100 \mathrm{~Hz}$. An internal low-pass filter was used with a cut-off frequency of $15 \mathrm{~Hz}$. This low-pass filter reduced the noise of the sensor from \pm 10 counts to \pm 1 count.

A script in Matlab segmented the raw data into each step portion that remained relatively constant. Each constant section of data was averaged and plotted as a single point. An example of raw data for compressive force applied in
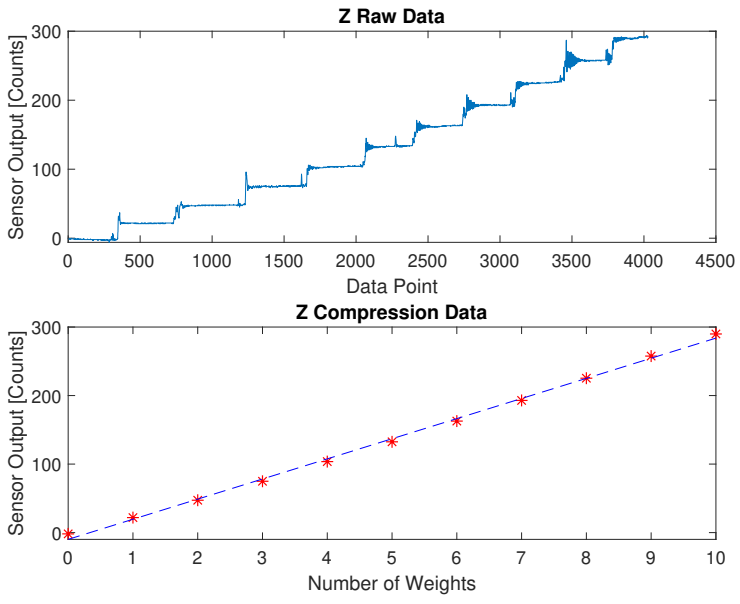

Fig. 3. Raw data and point data for compressive force in z-axis

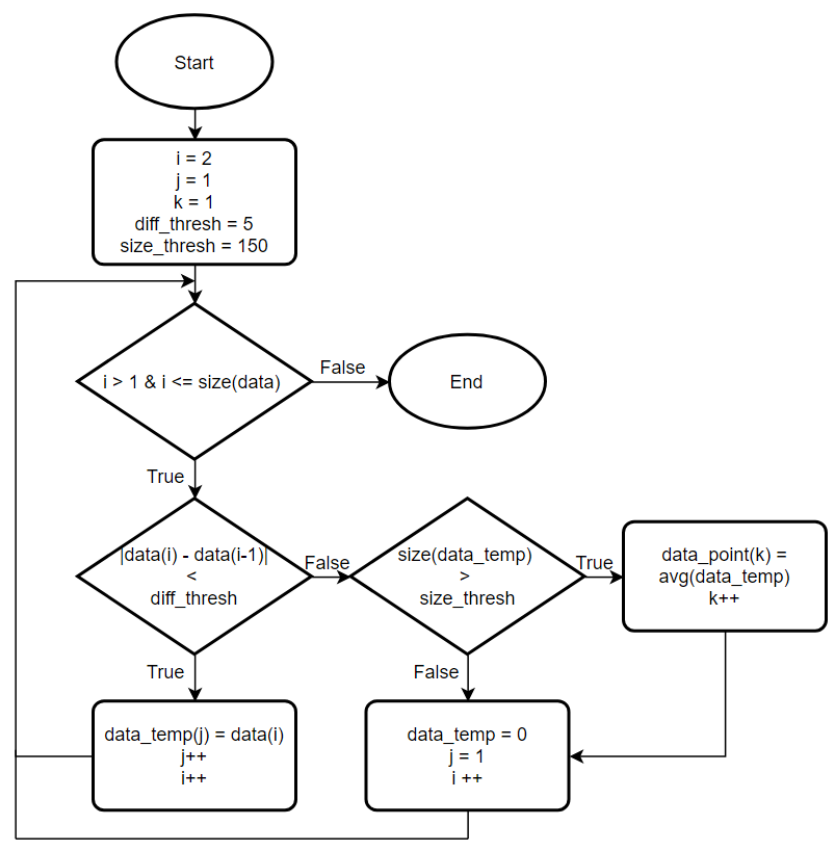

Fig. 4. Flowchart for the code to segment the raw data

the $\mathrm{z}$-axis and the resulting segmented point data is shown in Fig. 3. A flowchart for the code that performed this process to convert 'data' to 'data_point' is shown in Fig. 4. The difference threshold, 'diff_thresh', was chosen as 5 counts to account for noise and small vibrations that the sensor picked up during the constant force segments. The size threshold, 'size_thresh', was chosen as 150 data points so that brief portions of the constant force were not mistaken as data points.

\section{Main Results}

\section{A. Calibration}

To determine the calibration factor for the sensor in each axis, a linear line was fit to the 11 points of experimental data. The slope of the line is considered as the calibration 


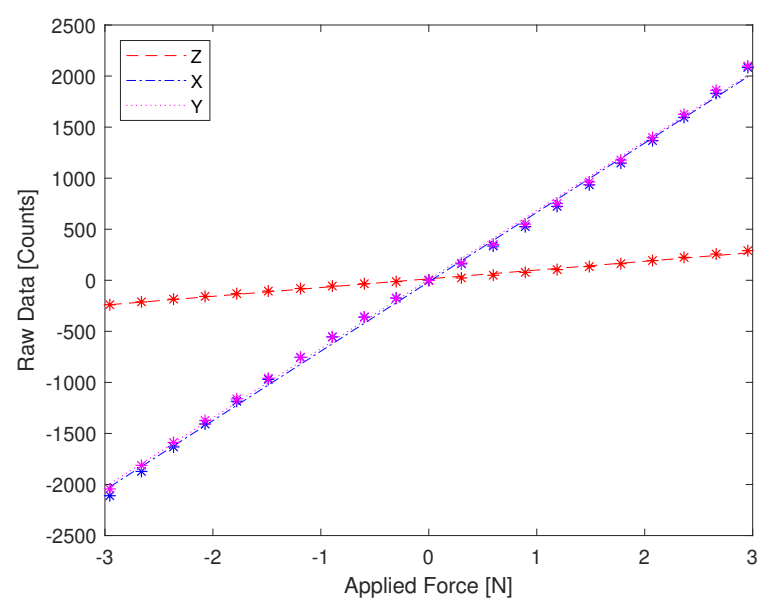

Fig. 5. Sensor data and linear fit calibration curves for $\mathrm{x}, \mathrm{y}$ and $\mathrm{z}$-axes

TABLE II

EXPERIMENTAL CALIBRATION DATA FOR X, Y AND Z-AXES

\begin{tabular}{cccc}
\hline & $\mathrm{Z}$ & $\mathrm{X}$ & $\mathrm{Y}$ \\
\hline Sensitivity [Counts/N] & 85.7 & 679.7 & 677.2 \\
Resolution [N/Count] & 0.012 & 0.0015 & 0.0015 \\
Zero Offset [N] & 0.17 & -0.022 & -0.011 \\
\hline
\end{tabular}

factor to convert the raw sensor count data into Newtons. The resolutions of the sensor are defined as the reciprocals of the calibration factors. The sensor data and calibration curves for each axis are plotted in Fig. 5. The sensitivities, resolutions, and zero offsets are presented in Table II. The maximum zero offset is in the z-axis and is $5.73 \%$ of the working range of $\pm 2.95 \mathrm{~N}$. A source of error in calculating the calibration may be that the sensor was not loaded to its nominal capacities.

The sensor has been calibrated twice before. Once in 2016 by the manufacturer and once in 2017 by a user in [6]. The sensitivity of the sensor is plotted over time in Fig. 6. The expected sensitivity, given by the manufacturer specification sheet, is also plotted. Note that in 2016 the sensor was calibrated to nominal capacity whereas in 2017 the sensor was calibrated with loads ranging from $-5 \mathrm{~N}$ to $8 \mathrm{~N}$ [6].

The differences between the measured and applied forces are plotted in Fig. 7 using the calibration data from Table II. The shape of the differences is consistent for the three axes. The average and maximum differences between the measured and applied forces are shown in Table III. Note that the values in this table are calculated based on the absolute differences.

\section{B. Hysteresis}

The hysteresis of the sensor is measured as the maximum difference between the readings of loading and unloading the sensor. The maximum percentage differences are shown in Table IV with respect to the working range of $2.95 \mathrm{~N}$ and with respect to the nominal capacities of the sensor. The maximum deviation with respect to the working range gives an accurate assessment of the non-linearity of the sensor for low

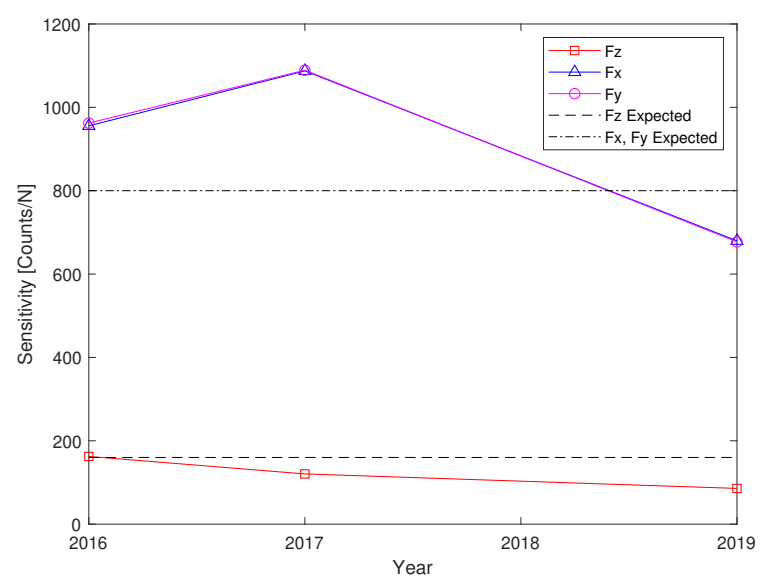

Fig. 6. Sensor sensitivity over time

TABLE III

DIFFERENCES BETWEEN MEASURED FORCES AND APPLIED FORCES FOR $X, Y$ AND Z-AXES

\begin{tabular}{cccc}
\hline & $\mathrm{Z}$ & $\mathrm{X}$ & $\mathrm{Y}$ \\
\hline Average Deviation [N] & 0.17 & 0.070 & 0.057 \\
Maximum Deviation [N] & 0.43 & 0.15 & 0.14 \\
\hline
\end{tabular}

forces. The maximum deviation with respect to the nominal capacities gives an approximate assessment of the full-scale non-linearity that can be compared to the specifications given by the manufacturer in Table I.

The average sensor readings for the hysteresis trials and the absolute differences between loading and unloading are plotted in Fig. 8 for compression and tension in the z-axis. The magnitude of sensor readings in unloading is higher than loading in both directions. The magnitude and quadratic shape of the differences is consistent for both directions.

\section{Repeatability}

The sensor outputs varied between each test for each applied load. The repeatability of the sensor is quantified as the sample standard deviation of the data:

$$
S_{j}=\sqrt{\frac{\sum_{i=1}^{n}\left(\bar{Y}_{j}-Y_{j i}\right)^{2}}{n-1}},
$$

where $S_{j}$ is the sample standard deviation, $\bar{Y}_{j}$ is the mean of the measured data, $Y_{j i}$ is the measured data, $n$ is the total number of tests, $j$ is the data point number, and $i$ is the test number. The average standard deviation and maximum standard deviation of each axis are shown in Table V.

The plots of the measured data for five trials in each axis and the standard deviation data are shown in Fig. 9. The differences between the trials approaches $0 \mathrm{~N}$ as the applied force approaches $0 \mathrm{~N}$. The parabolic shape of the standard deviation data is consistent for all axes except for the positive direction of the $\mathrm{x}$-axis and the maximum applied force in the $\mathrm{z}$-axis. In the positive direction $\mathrm{x}$-axis, the sensor readings are 

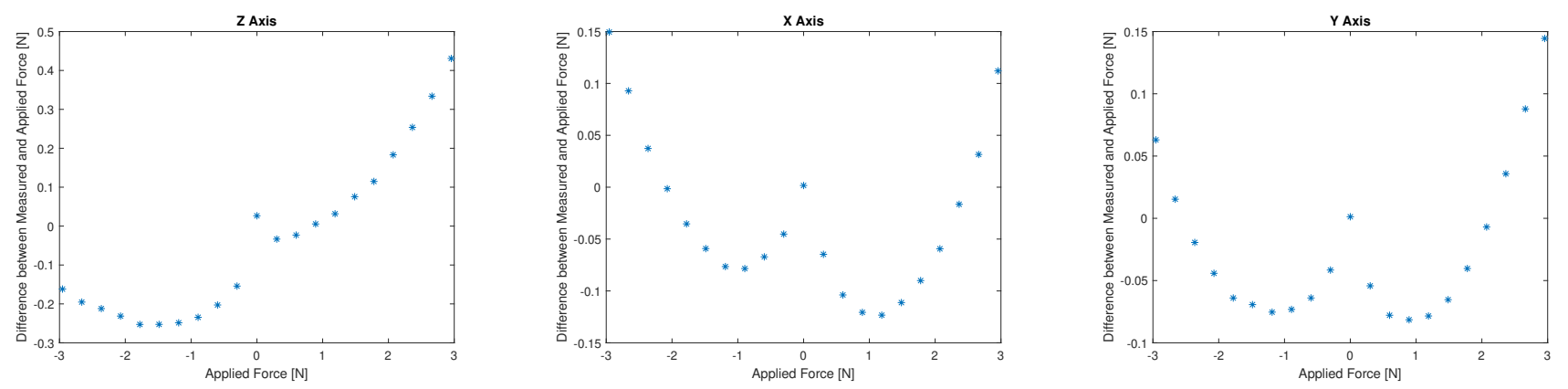

Fig. 7. Differences between applied and measured forces for $\mathrm{x}, \mathrm{y}$ and $\mathrm{z}$-axes
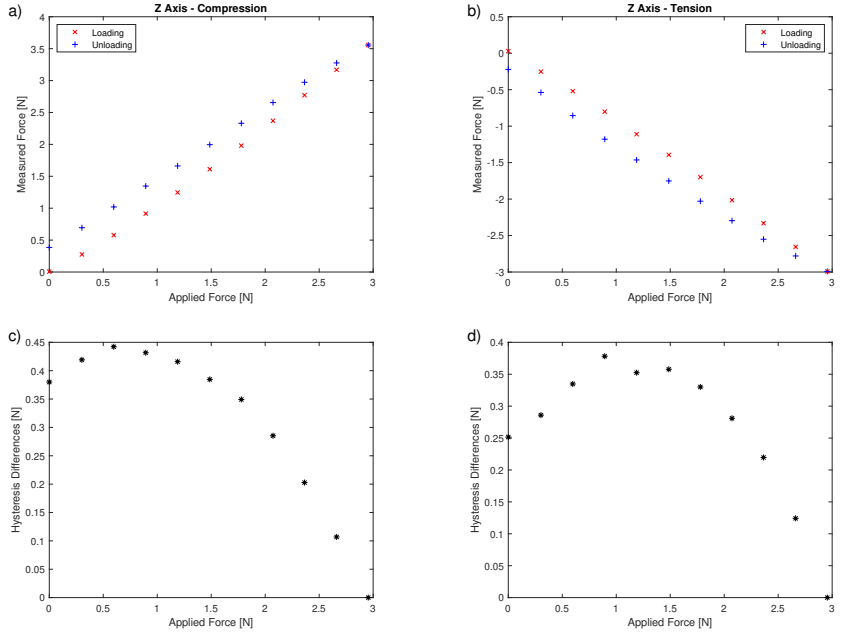

Fig. 8. (a),(b) Average measurement data for loading and unloading in the $\mathrm{z}$-axis. (c),(d) Absolute difference data for loading and unloading in the $\mathrm{z}$-axis.

TABLE IV

MAXIMUM HYSTERESIS PERCENTAGE DIFFERENCES WITH RESPECT TO THE WORKING RANGE (W.R.) AND THE NOMINAL CAPACITIES (N.C.)

\begin{tabular}{lcc}
\hline & $\mathrm{Z}_{\text {comp }}$ & $\mathrm{Z}_{\text {tens }}$ \\
\hline Max Difference W.R. & $15.0 \%$ & $12.8 \%$ \\
Max Difference N.C. & $0.4 \%$ & $0.8 \%$ \\
\hline
\end{tabular}

consistent between trials. At the maximum applied force in the z-axis, the standard deviation data starts to level out. The magnitude of the standard deviation data is significantly higher in the $\mathrm{z}$-axis, by a factor of $\sim 2$ than the $\mathrm{x}$-axis and a factor of $\sim 4$ than the $y$-axis.

\section{Non-Linearity}

The non-linearity of the sensor is quantified as the maximum deviation between the sensor reading and the linear line of best fit. The equation of the maximum percentage deviation from linearity is:

$$
\% d=100 \times \frac{\left|Y_{i}-M \times X_{i}\right|_{\max }}{M \times X_{f r}},
$$

where $Y_{i}$ is the measured data, $M$ is the slope of the linear line of best fit, $X_{i}$ is the applied force, and $X_{f r}$ is the full
TABLE V

AVERAGE AND MAXIMUM STANDARD DEVIATION BETWEEN 5 TRIALS

\begin{tabular}{cccc}
\hline & $\mathrm{Z}$ & $\mathrm{X}$ & $\mathrm{Y}$ \\
\hline Average Standard Deviation [N] & 0.091 & 0.034 & 0.040 \\
Maximum Standard Deviation [N] & 0.19 & 0.11 & 0.068 \\
\hline
\end{tabular}

TABLE VI

MAXIMUM DEVIATIONS FROM LINEAR LINES OF BEST FIT WITH RESPECT TO THE WORKING RANGE (W.R.) AND THE NOMINAL CAPACITIES (N.C.)

\begin{tabular}{lcccc}
\hline & $\mathrm{Z}_{\text {comp }}$ & $\mathrm{Z}_{\text {tens }}$ & $\mathrm{X}$ & $\mathrm{Y}$ \\
\hline Max Deviation W.R. & $14.6 \%$ & $8.6 \%$ & $3.8 \%$ & $4.9 \%$ \\
Max Deviation N.C. & $0.4 \%$ & $0.5 \%$ & $1.1 \%$ & $1.4 \%$ \\
\hline
\end{tabular}

working range of the applied force. The maximum percentage deviations from linearity for each axis are shown in Table VI. The table contains the maximum deviations with respect to the working range of $2.95 \mathrm{~N}$ and with respect to the nominal capacities of the sensor. The maximum deviation with respect to the working range gives an accurate assessment of the nonlinearity of the sensor for low forces. The maximum deviation with respect to the nominal capacities gives an approximate assessment of the full-scale non-linearity that can be compared to the specifications given by the manufacturer in Table I.

The average sensor readings from the five trials, the linear lines of best fit and the residuals of each axis are plotted in Fig. 10. The cubic shape of the residuals is consistent for all axes. The maximum magnitude of the residuals occurs at the max loading in the positive direction for all axes.

\section{DISCUSSION}

According to [6], the required resolution for a sensor used to validate a haptic device is $0.1 \mathrm{~N}$. The lowest resolution of the optical force sensor is $0.012 \mathrm{~N}$ in the $\mathrm{z}$-axis. Therefore, the sensitivity of the sensor meets the requirement. The zero offsets of the linear lines of best fit are relatively small, the highest being $5.73 \%$ of the working range and can be considered negligible.

Compared to the manufacturer resolution specifications in Table I, there is a $15 \%$ drop in the $\mathrm{x}$ and y-axes and a $46 \%$ drop in the z-axis. It is clear from Fig. 6 that the sensitivity of the sensor changes significantly over time. This indicates 

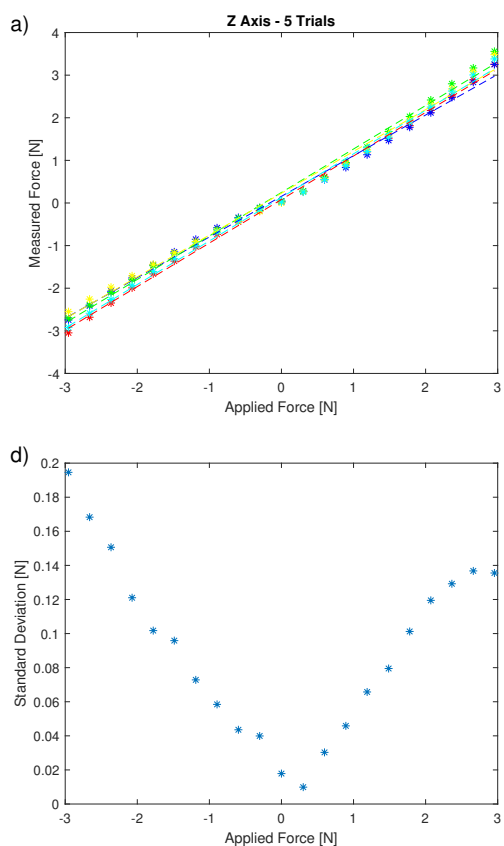
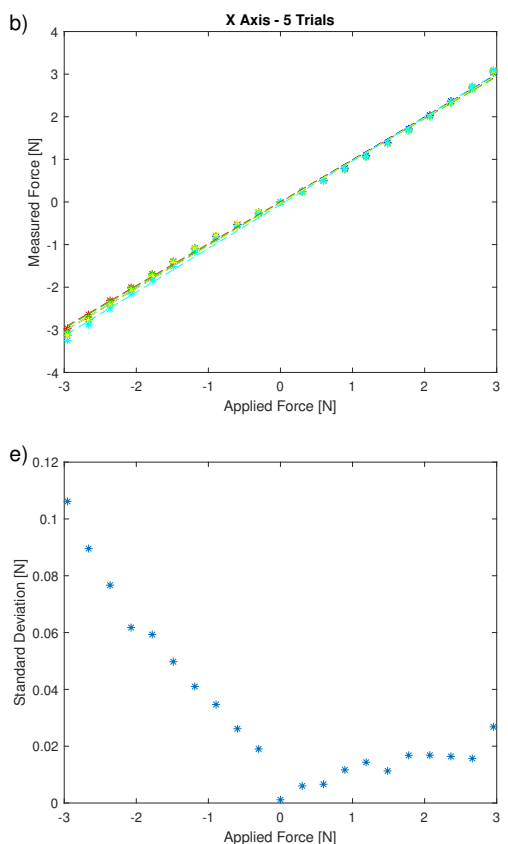
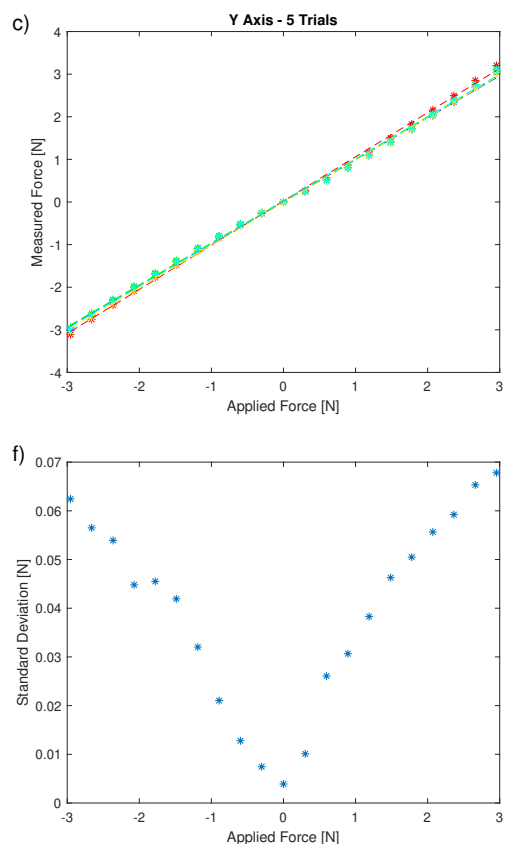

Fig. 9. (a)-(c) Measurement data for 5 trials in $x, y$, and z-axes. (d)-(f) Standard deviation data for 5 trials in $x$, $y$, and $z$-axes.

that the sensor must be calibrated between uses. It can also be observed that the sensitivity is declining over time. This implies that the optical force sensor has a lifetime before the sensitivity reduces to an inadequate level.

From [6], the required accuracy for the sensor is $0.5 \mathrm{~N}$. The maximum deviation of the sensor reading from the applied force is $0.43 \mathrm{~N}$ in the $\mathrm{z}$-axis. Therefore, from the experimental data, the accuracy of the sensor meets the requirement. The trends of the accuracy in Fig. 7 indicates that past $2 \mathrm{~N}$ the accuracy will decrease as the magnitude of the applied force increases.

From the standard deviation data, it can be observed that as the magnitude of the force increases, the variation between trials increases and the repeatability of the sensor decreases. It is also observed that the repeatability is significantly worse in the $\mathrm{z}$-axis than the $\mathrm{x}$ and $\mathrm{y}$-axes. The maximum standard deviation is $0.19 \mathrm{~N}$ in the $\mathrm{z}$-axis, which is an acceptable level for low force applications.

From the hysteresis data in Fig. 8, the magnitude of the sensor readings is higher in unloading than in loading. The parabolic shape of the difference data indicates that the sensor has a significant hysteresis effect. It is notable that there is a residual force reading of an average of $9.4 \%$ of the maximum load once the applied force is unloaded back to $0 \mathrm{~N}$. This may be due to the physical properties of the sensor. The sensing surface is made of a rubber material that may not instantly return to its original shape after a load is removed. This would result in a false force reading from the residual deformation of the material. The max deviation from loading to unloading with respect to the nominal capacity is $0.8 \%$ and agrees with the manufacturer specifications in Table I.

The sensor demonstrates some non-linearity. The maximum deviation from linearity with respect to the nominal capacity is $1.4 \%$ in the $y$-axis and agrees with the manufacturer specification in Table I. The cubic trend of the residuals in Fig. 10 indicate that there is a constant non-linearity effect across all axes of the sensor.

For the hysteresis and non-linearity, the results with respect to the working range are higher by factors of up to 20 than the results with respect to the nominal capacities. Testing the hysteresis and non-linearity to nominal capacity may result in higher relative inaccuracies which may exceed the specifications given by the manufacturer. Additionally, the sensor may also experience crosstalk and drift, which should be analysed to identify additional sources of inaccuracies.

\section{Conclusions}

The optical force sensor was calibrated, and the repeatability, hysteresis, and non-linearity of the sensor were observed. The experimental results agree with the specifications given by the manufacturer and the sensor meets the requirements for validating a haptic system, as defined by [6]. It is clear from the hysteresis and non-linearity results that this sensor may not be suitable for all low force applications and that the data in this paper should be used to evaluate the sensor on a case by case basis. It was observed that the $\mathrm{z}$-axis performs worse than the $\mathrm{x}$ and $\mathrm{y}$-axes in sensitivity deterioration, accuracy, repeatability, and non-linearity. It is also notable that the sensor retains a $10 \%$ force reading after it is unloaded. It is recommended that the sensor is frequently calibrated so that the deterioration of the sensitivity can be observed, and an accurate lifetime of the sensor can be modelled. It is also recommended that the sensor is tested to its nominal capacity to observe the results for the full range of the sensor. It 

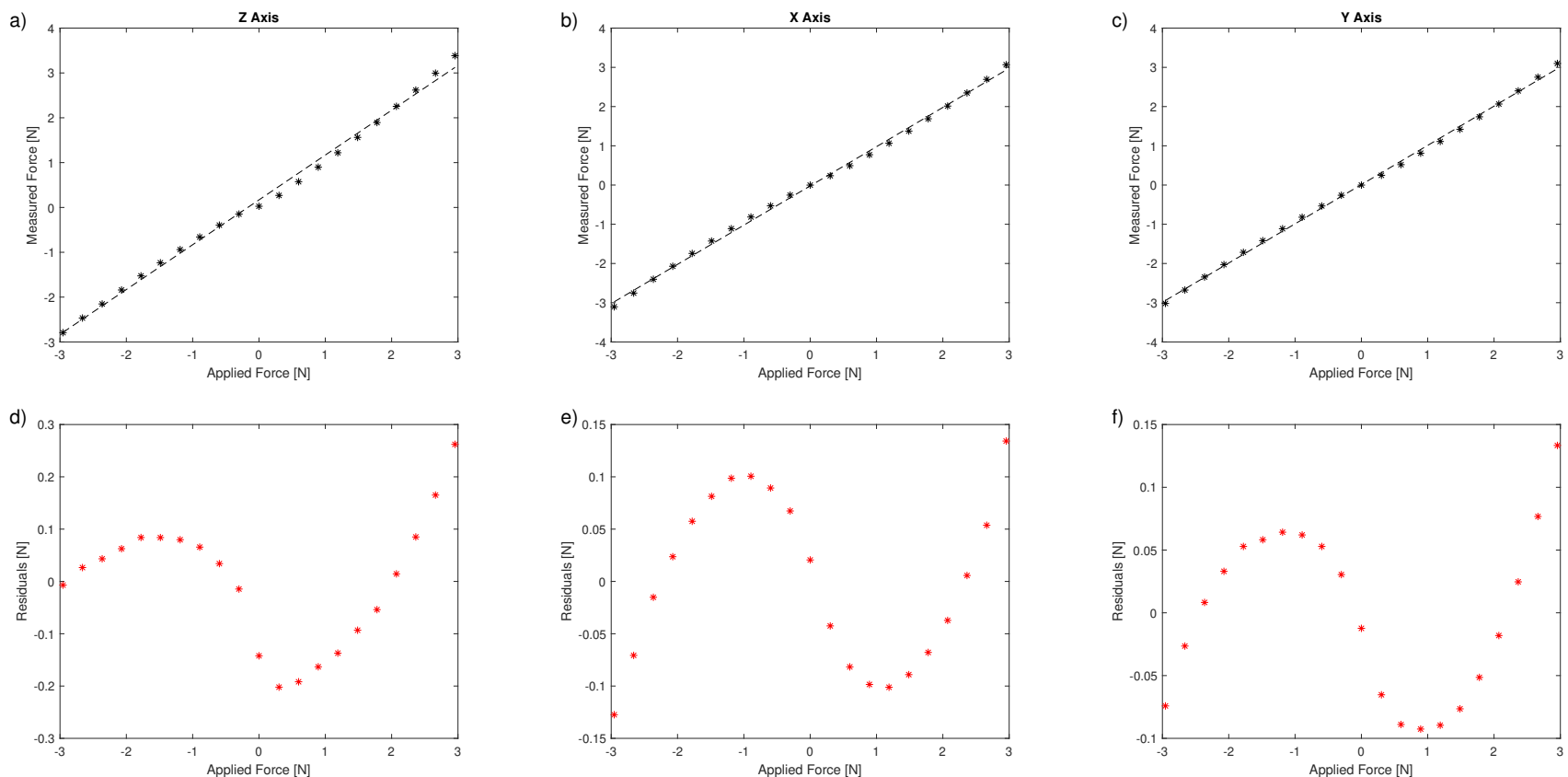

Fig. 10. (a)-(c) Average measurement data in $x, y$, and z-axes. (d)-(f) Residual data from linear line of best fit for $x, y$, and $z$-axes.

would be of interest to compare the results of this sensor to other types of force sensors to determine the advantages and disadvantages of optical force sensors.

\section{REFERENCES}

[1] A. Tar and G. Cserey, "Development of a low cost 3d optical compliant tactile force sensor," in 2011 IEEE/ASME International Conference on Advanced Intelligent Mechatronics (AIM), Budapest, Hungary, 2011, pp. 236-240.

[2] L. S. Lincoln, M. Quigley, B. Rohrer, C. Salisbury, and J. Wheeler, "An optical 3d force sensor for biomedical devices," in 2012 4th IEEE RAS \& EMBS International Conference on Biomedical Robotics and Biomechatronics (BioRob), Rome, Italy, 2012, pp. 1500-1505.

[3] M. Hossny, A. Bhatti, S. Nahavandi, and R. Tilove, "Force reflection distribution of haptic devices," in 2014 8th International Conference on Signal Processing and Communication Systems (ICSPCS), Gold Coast, QLD, Australia, 2014, pp. 1-9.

[4] G. Lee, S.-m. Hur, and Y. Oh, "A novel haptic device with high-force display capability and wide workspace," in 2016 IEEE International Conference on Robotics and Automation (ICRA), Stockholm, Sweden, 2016, pp. 2704-2709.

[5] J. Pfleging, M. Stücheli, R. Iovita, and J. Buchli, "Dynamic monitoring reveals motor task characteristics in prehistoric technical gestures," PloS one, vol. 10, no. 8, p. e0134570, 2015.

[6] S. Forbrigger, "Prediction-based haptic interfaces to improve transparency for complex virtual environments," Master's thesis, Dalhousie University, Halifax, 2017.

[7] S. Fekete, "Optoforce - senses for automation," 2017. [Online]. Available: http://donar.messe.de/exhibitor/hannovermesse/2017/Q631014/whowe-are-eng-494938.pdf

[8] Optoforce, "3-axis force sensor," OMD-20-FG-100N datasheet, 2016. 